\title{
RADII PROBLEMS FOR SECTIONS OF CONVEX FUNCTIONS
}

\author{
HERB SILVERMAN
}

(Communicated by Paul S. Muhly)

\begin{abstract}
A classical theorem of Szegö states that the sections $f_{n}(z)=$ $z+\sum_{k=2}^{n} a_{k} z^{k}$ of a convex function $f(z)=z+\sum_{k=2}^{\infty} a_{k} z^{k}$ must be convex for $|z|<\frac{1}{4}$. We determine disks $|z|<r_{n}$ in which $f_{n}$ is starlike and starlike of a positve order. Our proofs rely on some properties of convolutions.
\end{abstract}

1. Introduction. The convolution or Hadamard product of two power series $f(z)=\sum_{n=0}^{\infty} a_{n} z^{n}$ and $g(z)=\sum_{n=0}^{\infty} b_{n} z^{n}$ is defined as the power series

$$
(f * g)(z)=\sum_{n=0}^{\infty} a_{n} b_{n} z^{n} .
$$

Denote by $S^{*}$ the family consisting of functions $f(z)=z+\sum_{n=2}^{\infty} a_{n} z^{n}$ that are analytic and starlike in $\Delta=\{|z|<1\}$ and by $K$ the subfamily of functions convex in $\Delta$. In 1958 Pólya and Schoenberg conjectured in [3] that the convolution of two functions convex in $\Delta$ is also convex in $\Delta$. Since $f * g=h$ is equivalent to $f * z g^{\prime}=z h^{\prime}$, the well-known relationship $f \in K \Leftrightarrow z f^{\prime} \in S^{*}$ furnishes us with an alternate statement of the Pólya-Schoenberg conjecture. This conjecture was proved in 1973 by Ruscheweyh and Sheil-Small.

THEOREM A [5]. If $f \in K$ and $g \in K\left(g \in S^{*}\right)$, then $f * g \in K\left(f * g \in S^{*}\right)$.

Goodman and Schoenberg in [1] applied Theorem A to give a much simpler proof of the 1928 classical theorem of Szegö on the sections of convex functions.

THEOREM B [7]. If $f(z)=z+\sum_{k=2}^{\infty} a_{k} z^{k} \in K$, then $f_{n}(z)=z+\sum_{k=2}^{n} a_{k} z^{k}$ is convex in $|z|<\frac{1}{4}$ for all $n$ and $\frac{1}{4}$ is the largest radius with this property.

Goodman and Schoenberg first observed that it was sufficient to look at sections of the convex function $g(z)=\sum_{k=1}^{\infty} z^{k}=z /(1-z)$. For if $g_{n}(z)=z+\sum_{k=2}^{n} z^{k}$ is convex in $|z|<t<1$, then $g_{n}(t z) / t \in K$ and hence $h_{n}(z)=f(z) * g_{n}(t z) / t=$ $z+\sum_{k=2}^{n} a_{k} t^{k-1} z^{k} \in K$. Consequently, $t h_{n}(z / t)=f_{n}(z)$ is convex in $|z|<t$. Their proof was concluded when $t=\frac{1}{4}$ was shown.to be admissible.

An identical argument shows that any section of a convex function is starlike in $|z|<t$ whenever $g_{n}(z)$ is. In this note, we find disks $|z|<r_{n}$ in which the sections $f_{n}$ of a convex function $f$ are starlike. Analogous results are obtained for functions of positive order. Finally, we examine what can happen when the sections are replaced

Received by the editors June $8,1987$.

1980 Mathematics Subject Classification (1985 Revision). Primary 30C45; Secondary 30C50.

Key words and phrases. Univalent, starlike, convex, convolution. 
by more general functions containing infinitely many nonvanishing coefficients of $f$. A conjecture that would then generalize Szegö's Theorem B is given.

In the sequel we shall assume, unless otherwise stated, that $f(z)=z+\sum_{k=2}^{\infty} a_{k} z^{k}$ $\in K$, that $g(z)=z+\sum_{k=2}^{\infty} z^{k}$, and that $f_{n}$ and $g_{n}$ are the sections of $f$ and $g$ respectively.

\section{Radius of starlikeness for sections of $K$.}

THEOREM 1. If $f \in K$, then $f_{n}$ is starlike in $|z|<(1 / 2 n)^{1 / n}$ for all $n$.

PROOF. It suffices to prove the theorem for

$$
g_{n}(z)=z+\sum_{k=2}^{n} z^{k}=\left(z-z^{n+1}\right) /(1-z) .
$$

We have

$$
\frac{z g_{n}^{\prime}(z)}{g_{n}(z)}=\frac{1}{1-z}-\frac{n z^{n}}{1-z^{n}}
$$

so that for $|z|=r$,

$$
\operatorname{Re}\left\{\frac{z g_{n}^{\prime}(z)}{g_{n}(z)}\right\} \geq \frac{1}{1+r}-\frac{n r^{n}}{1-r^{n}}
$$

We need only show that the right-hand side of (2) is nonnegative or, equivalently, that

$$
1-(n+1) r^{n}-n r^{n+1} \geq 0
$$

for $r \leq(1 / 2 n)^{1 / n}$. But when $r=(1 / 2 n)^{1 / n},(3)$ is equivalent to $r=(1 / 2 n)^{1 / n} \leq$ $1-1 / n$, which is true for all $n \geq 2$.

Since the sequence $(1 / 2 n)^{1 / n}$ increases with $n$, a consequence of Theorem 1 is

COROLLARY 1. If $f \in K$, then $f_{n}$ is starlike in $|z|<\frac{1}{2}$ and $\frac{1}{2}$ is the largest radius with this property.

Note for $g_{2}(z)=z+z^{2}$ that $g_{2}^{\prime}(z)=1+2 z=0$ at $z=-\frac{1}{2}$, which means that the radius of univalence of sections of a convex function is also $\frac{1}{2}$.

COROLlaRY 2. If $f \in K$, then $f_{n}$ is starlike in $|z|<\sqrt{23 / 71} \approx 0.569$ for $n \geq 3$ and $\sqrt{23 / 71}$ is the largest radius with this property.

ProOF. From (1) we have for $z=r e^{i \theta}$,

$$
\begin{aligned}
\operatorname{Re}\left\{\frac{z g_{3}^{\prime}(z)}{g_{3}(z)}\right\} & =\operatorname{Re}\left\{\frac{1}{1-z}-\frac{3 z^{3}}{1-z^{3}}\right\}=\operatorname{Re}\left\{\frac{1+2 z+3 z^{2}}{1+z+z^{2}}\right\} \\
& =\frac{\left(1-2 r^{2}+3 r^{4}\right)+\left(3 r+5 r^{3}\right) \cos \theta+8 r^{2} \cos ^{2} \theta}{\left|1+z+z^{2}\right|^{2}} .
\end{aligned}
$$

For each $r$ the numerator of this last expression, which we denote by $h(r, \theta)$, has a minimum at $\theta=\pi$ or at

$$
\theta=\theta_{0}=\cos ^{-1}\left(-\frac{3+5 r^{2}}{16 r}\right) .
$$


Since $h(r, \pi) \geq 0$ for $0 \leq r \leq 1$ and $h\left(r, \theta_{0}\right)=\left(23-94 r^{2}+71 r^{4}\right) / 32=0$ for $r=\sqrt{23 / 71}$, the bound cannot be increased for $n=3$. The result follows from Theorem 1 upon observing for $n \geq 4$ that $(1 / 2 n)^{1 / n} \geq(1 / 8)^{1 / 4}>(23 / 71)^{1 / 2}$.

REMARKS. For $n$ even, we may set $z=-r$ in (1) to conclude that the radius of starlikeness of $g_{n}$ is the unique positive $r$ for which equality holds in (3). There does not appear to be such a "nice" polynomial equation from which to determine the radius of starlikeness of sections of a convex function when $n$ is odd.

In 1941, Robertson proved [4] that sections of a function in $S^{*}$ must be starlike in $|z|<1-(4 \log n) / n$ for $n>N$. He further showed that sections of the Koebe function $z /(1-z)^{2}$ are starlike in $|z|<1-(3 \log n) / n$ and that the constant 3 cannot be lowered. But $f$ is convex whenever $z f^{\prime}$ is starlike, so that we may translate Robertson's result to its convex analog. In view of the more recent developments (Theorem A), Robertson essentially proved

THEOREM 2. If $f \in K$, then $f_{n}$ is convex in $|z|<1-(3 \log n) / n$ for $n$ sufficiently large. The constant 3 cannot be lowered.

REMARK. Since

$$
\left(1-\frac{a \log n}{n}\right)^{n} \sim \frac{1}{n^{a}} \quad(n \rightarrow \infty),
$$

we see for any $a>1$ and $n$ sufficiently large that $(1 / a n)^{1 / n}>1-(a \log n) / n$. To put Theorem 1 in a form analogous to Theorem 2, we have for any $\varepsilon>0$ and $n>N(\varepsilon)$ that $f_{n}$ is starlike in $|z|<1-((1+\varepsilon) \log n) / n$.

3. Positive order. Denote by $S^{*}(\alpha), 0 \leq \alpha<1$, the family consisting of analytic functions $f(z)=z+\cdots$ that satisfy $\operatorname{Re}\left\{z f^{\prime} / f\right\}>\alpha, z \in \Delta$, and by $K(\alpha)$ the subfamily for which $\operatorname{Re}\left\{1+z f^{\prime \prime} / f^{\prime}\right\}>\alpha, z \in \Delta$. Such functions are said to be, respectively, starlike of order $\alpha$ and convex of order $\alpha$. The work of Ruscheweyh and Sheil-Small [5] actually shows that if $g$ in Theorem A is in $K(\alpha)$ or $S^{*}(\alpha)$, then so is $f * g$.

The first important result about functions of positive order is due to Marx [2] and Strohhäcker [6] who independently proved that $K \subset S^{*}\left(\frac{1}{2}\right)$. In fact, they showed that $\operatorname{Re}\left\{z f^{\prime} / f\right\} \geq 1 /(1+r), f \in K$, with equality for $f(z)=z /(1-z)$ at $z=-r$. We may thus conclude that $f$ in $K$ is starlike of order $\alpha$ for $|z|<(1-\alpha) / \alpha$ when $\frac{1}{2} \leq \alpha<1$. The more general form of the theorem of Ruscheweyh and Sheil-Small will now be applied to find disks in which sections of $f$ are starlike of order $\alpha$.

THEOREM 3. If $f \in K$, then $f_{n}$ is starlike of order $\alpha$ in $|z|<(1-\alpha) /(2-\alpha)$ and $(1-\alpha) /(2-\alpha)$ is the largest radius with this property.

ProOF. In view of (2), it suffices to show that

$$
\frac{1}{1+r}-\frac{n r^{n}}{1-r^{n}} \geq \alpha
$$

for $r \leq(1-\alpha) /(2-\alpha)$. Since equality in (5) holds for $r=(1-\alpha) /(2-\alpha)$ and $n=2$, we need only show that $n r^{n} /\left(1-r^{n}\right)$ is a decreasing function of $n$ for $r=(1-\alpha) /(2-\alpha)$. But $(n+1) r^{n+1} /\left(1-r^{n+1}\right) \leq n r^{n} /\left(1-r^{n}\right)$ is equivalent to $n-(n+1) r+r^{n+1} \geq 0$, which follows upon noting that the left-hand side is a decreasing function of $r(\leq 1)$. The function $g_{2}(z)=z+z^{2}$ is extremal. 
In light of the Marx and Strohhäcker result, it is no surprise that the value $\frac{1}{2}$ plays a distinguishing role when finding disks for which sections of convex functions are starlike of order $\alpha$. We show that the $f_{n}$ must be starlike of order $\alpha$ in disks $|z|<r_{n} \rightarrow 1$ if and only if $\alpha \leq \frac{1}{2}$.

THEOREM 4. Suppose $f \in K$.

(i) If $0 \leq \alpha<\frac{1}{2}$, then $f_{n}$ is starlike of order $\alpha$ in $|z|<((1-2 \alpha) / 2 n)^{1 / n}$ for all $n$.

(ii) The function $f_{n}$ is starlike of order $\frac{1}{2}$ in $|z|<\left(4 / 9 n^{2}\right)^{1 / n}$ for all $n$.

(iii) If $\frac{1}{2}<\alpha<1$, then $g_{2 n}$ is not starlike in $|z|<(1-\alpha) / \alpha<1$ for any $n$.

PROOF. We rewrite (5) as

$$
(1-\alpha)-\alpha r-(n+1-\alpha) r^{n}-(n-\alpha) r^{n+1} \geq 0 .
$$

To prove (i), set $r=((1-2 \alpha) / 2 n)^{1 / n}$ in (6) to obtain

$$
r=\left(\frac{1-2 \alpha}{2 n}\right)^{1 / n} \leq \frac{n-(1-\alpha)(1-2 \alpha)}{n-\alpha(1-2 \alpha)}
$$

which is equivalent to

$$
h(\alpha, n)=\left(\frac{n-(1-\alpha)(1-2 \alpha)}{n-\alpha(1-2 \alpha)}\right)\left(\frac{2 n}{1-2 \alpha}\right)^{1 / n} \geq 1
$$

Since $h(\alpha, n) \geq h(0, n)=(1-1 / n)(2 n)^{1 / n} \geq h(0,2)=1$, (i) follows. Setting $\alpha=\frac{1}{2}$ and $r=\left(4 / 9 n^{2}\right)^{1 / n}$ in (6) leads to $4 / 9 n^{2} \leq\left(1-16 n /\left(9 n^{2}+8 n-4\right)\right)^{n}$, which is true for all $n$ with equality when $n=2$. This proves (ii). Finally, if $\frac{1}{2}<\alpha<1$ then the left-hand side of $(6)$ is less than $1-\alpha-\alpha r$, which vanishes for $r=(1-\alpha) / \alpha$.

REMARKS. While not sharp for every $n$, Theorem 4 is at least of the right order of magnitude. For $r=(A / n)^{1 / n}, A>(1-2 \alpha) / 2$, we may let $n \rightarrow \infty$ in (6) to get $1-2 \alpha-2 A<0$. Hence $g_{2 n}$ is not starlike of order $\alpha\left(<\frac{1}{2}\right)$ for $n$ sufficiently large. Similarly, we can show that $g_{2 n}$ is not starlike of order $\frac{1}{2}$ for $n$ large when $|z|<\left(\varepsilon / n^{\beta}\right)^{1 / n}, \varepsilon>0$ and $\beta<2$.

Turning now to disks in which sections of convex functions are convex of order $\alpha$, we note first that $\operatorname{Re}\left\{1+z f^{\prime \prime} / f^{\prime}\right\} \geq(1-r) /(1+r), f \in K$, so that $f$ is convex of order $\alpha$ when $|z|<(1-\alpha) /(1+\alpha)$. The next theorem reduces to Szegö's Theorem when $\alpha=0$. The proof uses the methods of Goodman and Schoenberg [1].

THEOREM 5. If $f \in K$, then $f_{n}$ is convex of order $\alpha$ in $|z|<(1-\alpha) / 2(2-\alpha)$ and $(1-\alpha) / 2(2-\alpha)$ is the largest radius with this property.

PROOF. For $g_{n}(z)=\left(z-z^{n+1}\right) /(1-z)$, we have

$$
1+\frac{z g_{n}^{\prime \prime}(z)}{g_{n}^{\prime}(z)}=\frac{n(n+1) z^{n}(z-1)}{1-(n+1) z^{n}+n z^{n+1}}+\frac{1+z}{1-z} .
$$

It suffices to show, for $|z|=(1-\alpha) / 2(2-\alpha)$, that

$$
\left|\frac{n(n+1) z^{n}(z-1)}{1-(n+1) z^{n}+n z^{n+1}}\right| \leq \operatorname{Re}\left\{\frac{1+z}{1-z}\right\}-\alpha .
$$


Since the right-hand side of (7) is minimized when $z$ is real and negative, we need only show that

$$
\frac{n(n+1)|z|^{n}(|z|+1)}{1-(n+1)|z|^{n}-n|z|^{n+1}} \leq \frac{3-\alpha}{5-3 \alpha}-\alpha=\frac{3(1-\alpha)^{2}}{5-3 \alpha} .
$$

Substituting in for $|z|$, a computation shows that (8) is equivalent to

$$
\frac{(5-3 \alpha)^{2}}{6(1-\alpha)^{2}(2-\alpha)} \leq \frac{2^{n}(2-\alpha)^{n}}{n(n+1)(1-\alpha)^{n}}-\frac{1}{n}-\frac{1-\alpha}{2(n+1)(2-\alpha)}=j(\alpha, n) .
$$

The result follows upon noting that $j(\alpha, n)$ is an increasing function of $n, \alpha$ fixed, with $j(\alpha, 2)=(5-3 \alpha)^{2} / 6(1-\alpha)^{2}(2-\alpha)$. The function $g_{2}(z)$ is extremal.

4. More general sequences. If $f(z)=z+\sum_{k=2}^{\infty} a_{k} z^{k} \in K$ and $\left\{a_{k_{j}}\right\}$ is any subsequence of $\left\{a_{k}\right\}$, finite or infinite, Theorem $\mathrm{A}$ may again be applied to show that $F_{k}(z)=z+\sum_{j=1}^{\infty} a_{k_{j}} z^{k^{j}}$ is starlike or convex whenever $G_{k}(z)=z+\sum_{j=1}^{\infty} z^{k^{j}}$ is. In the special case that $k_{j}=k$ for $1 \leq j \leq n(n=2,3, \ldots)$, our functions reduce to the sections previously investigated. To solve extremal problems, it behooves us to find as much information as possible about $G_{k}$. To illustrate with an example, if we set $k_{j}=j k+1$ then $G_{k}(z)=z+\sum_{j=1}^{\infty} z^{j k+1}=z /\left(1-z^{k}\right)$. This leads to

THEOREM 6. If $f(z)=z+\sum_{k=2}^{\infty} a_{k} z^{k} \in K$, then

$$
F_{k}(z)=z+\sum_{j=1}^{\infty} a_{j k+1} z^{j k+1} \quad(k=2,3, \ldots)
$$

is starlike in $|z|<(1 /(k-1))^{1 / k}$. The bound is sharp for every $k$.

PROOF. For $G_{k}(z)=z /\left(1-z^{k}\right)$, we have

$$
\begin{aligned}
\operatorname{Re}\left\{\frac{z G_{k}^{\prime}(z)}{G_{k}(z)}\right\} & =\operatorname{Re}\left\{\frac{1+(k-1) z^{k}}{1-z^{k}}\right\}=\frac{1+(k-2) r^{k} \cos k \theta-(k-1) r^{2 k}}{\left|1-z^{k}\right|^{2}} \\
& \geq \frac{1-(k-2) r^{k}-(k-1) r^{2 k}}{\left|1-z^{k}\right|^{2}} .
\end{aligned}
$$

Since the numerator of this last expression vanishes when $r=(1 /(k-1))^{1 / k}$, the proof is complete.

Now $(1 /(k-1))^{1 / k}$ attains its minimum when $k=5$. So with the notation of the theorem, we obtain

COROLlaRY 1. If $f \in K$, then $F_{k}$ is starlike in $|z|<(1 / 4)^{1 / 5} \approx 0.758$ for all $k=2,3, \ldots$.

One can obtain similar results about convexity. For instance, a simple computation shows the radius of convexity of $G_{2}(z)=z /\left(1-z^{2}\right)$ to be $\sqrt{2}-1$, which yields

CORLLARY 2. If $f(z)=z+\sum_{k=2}^{\infty} a_{k} z^{k} \in K$, then $F_{2}(z)=z+\sum_{j=1}^{\infty} a_{2 j+1} z^{2 j+1}$ is convex in $|z|<\sqrt{2}-1 \approx 0.414$.

The function $g_{2}(z)=z+z^{2}$ has been extremal for most of our results on sections of a convex function. We close with a conjecture that would establish $g_{2}$ as extremal in a more general setting, which incorporates Szegö's Theorem B and Corollary 1 of Theorem 1 as special cases. 
CONJECTURE. If $z+\sum_{k=2}^{\infty} a_{k} z^{k} \in K$ and $\left\{a_{k_{j}}\right\}$ is any (finite or infinite) subsequence of $\left\{a_{k}\right\}$, then $z+\sum_{j=1}^{\infty} a_{k_{j}} z^{k^{j}}$ is convex in $|z|<\frac{1}{4}$ and starlike in $|z|<\frac{1}{2}$.

\section{REFERENCES}

1. A. W. Goodman and I. J. Schoenberg, On a theorem of Szegö on univalent convex maps of the unit circle, J. Analyse Math. 44 (1984/85), 200-204.

2. A. Marx, Untersuchungen über Schlichte Abbildungen, Math. Ann. 107 (1932/33), 40-67.

3. G. Pólya and I. J. Schoenberg, Remarks on de la Vallée Poussin means and convex conformal maps of the circle, Pacific J. Math. 8 (1958), 295-334.

4. M. S. Robertson, The partial sums of multivalently star-like functions, Ann. of Math. 42 (1941), 829-838.

5. St. Ruscheweyh and T. Sheil-Small, Hadamard products of schlicht functions and the PólyaSchoenberg conjecture, Comment Math. Helv. 48 (1973), 119-135.

6. E. Strohhäcker, Beiträge zur Theorie der schlichten Funktionen, Math. Z. 37 (1933), 356-380.

7. G. Szegö, Zur Theorie der schlichten Abbildungen, Math. Ann. 100 (1928), 188-211.

Department of Mathematics, College of Charleston, Charleston, South CAROLINA 29424 